\title{
tic\&société
}

Vol. 9, No 1-2 | 1 er semestre 2015 - 2ème semestre 2015

Les religions au temps du numérique

\section{Une webcam à la grotte : Le sanctuaire marial de Lourdes et l'introduction des TNIC}

\section{Paul BERNADOU and Sylvaine GUINLE-LORINET}

\section{(2) OpenEdition}

\section{Journals}

Electronic version

URL: http://journals.openedition.org/ticetsociete/1880

DOI: 10.4000/ticetsociete.1880

\section{Publisher}

Association ARTIC

\section{Electronic reference}

Paul BERNADOU and Sylvaine GUINLE-LORINET, « Une webcam à la grotte : Le sanctuaire marial de Lourdes et l'introduction des TNIC », tic\&société [Online], Vol. 9, N 1-2 I 1er semestre 2015 - 2ème semestre 2015, Online since 11 January 2016, connection on 19 April 2019. URL : http:// journals.openedition.org/ticetsociete/1880 ; DOI : 10.4000/ticetsociete.1880 
tic\&société - 9 (1-2), 2015

Une webcam à la grotte : le sanctuaire marial de Lourdes et l'introduction des TNIC

\author{
Paul BERNADOU \\ Centre de recherches sur les médiations (Crem) \\ Université Paul-Verlaine \\ 18, rue Camille Saint-Saëns, 65000 Tarbes \\ paul.bernadou@orange.fr \\ Sylvaine GUINLE-LORINET \\ Université de Pau et des Pays de l'Adour \\ Identités, territoires, expressions, mobilités (Item) \\ 13, rue Miramont, 65000 Tarbes \\ sylvaine.guinle.lorinet@wanadoo.fr
}


Paul BERNADOU

Sylvaine GUINLE-LORINET

Une webcam à la grotte :

le sanctuaire marial de Lourdes et l'introduction des TNIC

Paul BERNADOU est docteur en sciences de l'information et de
la communication. II travaille depuis une quinzaine d'années sur
la communication du fait religieux et les rapports entre santé et
pratiques spirituelles autour du fait de Lourdes en particulier.
Ancré dans la posture ethnométhodologique, il a développé une
approche du fait religieux croisant les méthodes
ethnographiques de terrain et les analyses
communicationnelles classiques des médiations: discours,
images, corpus de bibliothèques.

Sylvaine GUINLE-LORINET, maître de conférences habilitée à diriger des recherches en histoire contemporaine, mène des recherches sur le catholicisme français très contemporain ; après avoir consacré une partie de ses travaux à l'histoire de Lourdes, elle s'est intéressée aux groupes marginaux ou contestataires de l'Église catholique. Elle travaille aussi sur la mémoire, notamment en milieu urbain, ses formes d'expression, ses liens avec le patrimoine culturel matériel ou/et immatériel.

Résumé : Existe-t-il un espace socialement partagé entre les pèlerins physiques et les utilisateurs du dispositif de TNIC mis à disposition par le sanctuaire marial de Lourdes ? L'analyse est conduite en s'appuyant sur l'histoire de la communication et sur une approche anthropologique de la réception du processus communicationnel du pèlerinage et des TNIC dans ce sanctuaire catholique. Nous proposons, dans une perspective anthropologique, de repérer les ressemblances et les différences entre les comportements des pèlerins " physiques » du sanctuaire et les internautes, ceci pourrait fournir, par inférence, des indications sur leurs espaces respectifs socialement partagés.

Mots-clés : sanctuaire, Lourdes, webpèlerinage, multimédia, histoire du fait religieux, anthropologie de la communication. 


\begin{abstract}
DICT and the Catholic religion: the example of Lourdes shrine. - Is there a social space shared both by physical pilgrims and users of the DICT network, put to their disposal by the Marian shrine? The analysis is informed by the history of communications and an anthropological approach to reception of the communicational process of pilgrimage and new communications technologies in this Catholic shrine. We propose an anthropological perspective to identify the similarities and differences between the behaviour of "physical" pilgrims to the shrine and Internet users. By inference, this could provide indications as to the spaces shared by these respective communities.
\end{abstract}

Keywords: Lourdes's marian shrine, web pilgrimage, multimedia, history of the religious fact, communications anthropology.

Resumen: Una webcam en la gruta. El santuario mariano de Lourdes y la introducción de las NTIC - ¿Existe un espacio socialmente compartido entre los peregrinos físicos y los usuarios del dispositivo NTIC puesto a su disposición por el santuario mariano de Lourdes? El análisis que aquí se propone se fundamenta en la historia de la comunicación y en un enfoque antropológico de la recepción del proceso comunicacional de la peregrinación y de las NTIC en dicho santuario católico. Desde una perspectiva antropológica, se propone identificar las semejanzas y las diferencias entre los comportamientoqs de los peregrinos "físicos " del santuario y Ios internautas; lo cual podría proporcionar, por inferencia, indicaciones sobre sus respectivos espacios socialmente compartidos.

Palabras clave: santuario mariano de Lourdes, webperegrinación, multimedia, historia del hecho religioso, antropología de la comunicación. 
En l'an 2000, les catholiques fêtent l'entrée dans le troisième millénaire de l'Incarnation du Christ (fils du dieu chrétien); dans le sanctuaire marial de Lourdes ${ }^{1}$, face à la grotte, une webcam ${ }^{2}$ est installée qui diffuse en quasi-direct sur le continent numérique les images du cœur de Lourdes. Dès ce moment les connexions au site internet du sanctuaire doublent, jusqu'à aujourd'hui, où le mouvement s'est amplifié, au point que la page de la webcam de la grotte (voir figure 1) est devenue le véritable portail internet du sanctuaire et l'un des sites web catholiques les plus consultés de France.

Dans cet article nous poursuivons par l'analyse de l'utilisation des TNIC une étude anthropologique de la réception du processus communicationnel du fait de Lourdes ${ }^{3}$. Depuis plus de cent cinquante ans, ce fait religieux a produit ce qui est reconnu comme un des premiers pèlerinages catholiques du monde. L'utilisation des TNIC dans le sanctuaire permet-elle d'étendre le pèlerinage de Lourdes au " continent numérique », comme le suggèrent les autorités du lieu ?

Anthropologue de la communication et historienne, nous associons nos démarches disciplinaires respectives : pour l'un, posture de l'ethnométhodologie, qui se définit comme la recherche empirique, par l'immersion objectivement reconnue dans la communauté, des logiques que les individus utilisent pour donner sens à leurs actions et les accomplir ; pour l'autre, posture classique de collecte, croisement et critique des sources, écrites et orales ${ }^{4}$, par l'historienne qui s'efforce de

\footnotetext{
1 Couvrant aujourd'hui un domaine de plus de cinquante hectares dans la partie basse de la ville, il s'est étendu en bordure du gave de Pau, à partir de la grotte de Massabielle. C'est précisément là qu'en 1858 éclate « l'affaire Lourdes » : une jeune fille de ce bourg pyrénéen, Bernadette Soubirous, déclare avoir vu quelque chose à la grotte, alors un endroit isolé près du gave. Les curieux se pressent ; les autorités civiles s'émeuvent, du maire au ministre de l'Intérieur, du procureur au garde des Sceaux, jusqu'à Napoléon III. En 1862, l'évêque catholique de Tarbes, Mgr Laurence, authentifie les apparitions mariales. Lourdes semble confirmer, aux yeux des autorités ecclésiastiques et des fidèles, le dogme de I'Immaculée Conception, par lequel, le 8 décembre 1854, dans la bulle Ineffabilis Deus, le pape Pie IX a déclaré Marie préservée du péché originel dès le premier instant de sa conception.

${ }^{2}$ Sanctuaire Notre-Dame de Lourdes, TV Lourdes, <http://fr.lourdes-france.org/tvlourdes/?v=newtvlourdes $>$, dernière consultation le 5 septembre 2015.

${ }^{3}$ Cette étude, commencée en 2002, comprenait dans un premier temps, une description ethnographique du pèlerinage "physique », puis de certaines de ses médiatisations (presse, photographies, bibliothèques).

${ }^{4}$ Les auteurs tiennent à remercier MM. Pierre Adias (ancien responsable du service communication du sanctuaire), Thierry Castillo (économe), Laurent Jarneau
} 
rester objective. Nous proposons d'expliquer la genèse de l'utilisation des TNIC dans le sanctuaire, de présenter le dispositif technique mis à disposition des internautes, puis nous questionnerons l'annexion du «continent numérique » qui semble être opérée par l'espace socialement partagé du pèlerinage.

\section{Du site internet au projet mix-media}

Bien que répondant au souhait général du texte du concile Vatican II consacré aux moyens de communication sociale (décret Inter mirifica, 1963, § 13), l'utilisation des TNIC dans le sanctuaire pourrait aussi être référée au chapitre numéro 37 de l'encyclique du pape Jean Paul II Redemptoris Missio, inspiré par Marshall McLuhan ${ }^{5}$. « II ne suffit pas d'utiliser les médias pour répandre le message chrétien et l'enseignement authentique de l'Église. " écrit en effet Pierre Babin ${ }^{6}$, " II est également nécessaire d'intégrer ce message dans la "nouvelle culture" engendrée par les communications modernes. " (Babin, 1999). L'utilisation des TNIC à Lourdes peut refléter ce passage du "message répandu" ${ }^{7}$ " à "l'intégration dans la nouvelle culture ». Ce passage s'est fait par tâtonnements et par développement méthodique suite à l'étape décisive de la webcam à la grotte de Massabielle à Lourdes.

(rédacteur en chef du site internet) et Mathias Terrier (responsable du pôle multimédia), qui ont répondu à leurs questions et leur ont fourni documents et statistiques, ainsi que le Père R. Boisvert (aumônier de l'Hospitalité Notre Dame de Lourdes).

${ }^{5}$ Si Marshall McLuhan (1911-1980) est bien connu en tant que théoricien canadien de la communication et des médias, il l'est moins pour sa foi catholique. II se convertit pourtant au catholicisme dans les années 1930. Dans les années 1970, mondialement connu, il devient conseiller du Vatican pour le Conseil pontifical des communications sociales. Son influence est sensible dans l'encyclique Redemptoris Missio (§ 37), dans laquelle Jean Paul II considère que pour la mission évangélique de l'Église catholique, " le premier aréopage des temps modernes est le monde de la communication, qui donne une unité à l'humanité en faisant d'elle, comme on dit, "un grand village". "

${ }^{6}$ Prêtre français, missionnaire oblat de Marie Immaculée, Pierre Babin (1925-2012) fut formé à la communication auprès de McLuhan et fonda le Centre de recherche et d'éducation en communication (Crec).

${ }^{7}$ M. Adias, ancien responsable du service communication du sanctuaire de Lourdes, atteste que lors de cette première étape « on a décliné des choses existantes [sur papier] sur internet » (entretien avec P. Adias, le 23 janvier 2014).

tic\&société - 9 (1-2), 2015 


\subsection{Des acteurs multiples}

Le premier site internet s'inscrit dans une politique de collaboration entre la ville de Lourdes et le sanctuaire, menée dès 1993, afin d'améliorer notamment l'accueil des foules et des malades. Cette réflexion s'est accompagnée de la création du site web, géré par une commission paritaire ville-sanctuaire depuis 1995.

À cette création ont travaillé, tant du côté de la ville que de celui du sanctuaire, des pionniers "politiques »: lorsque le premier site internet est créé, le P. Michel de Roton est recteur du sanctuaire. Aux journalistes auxquels il présente le nouveau site, il déclare être "croyant mais pas pratiquant " et la boutade est souvent répétée. Ignorant d'internet, le recteur a compris qu'il faut doter Lourdes de cet outil nouveau ${ }^{8}$. II donne donc le feu vert à une équipe formée de plusieurs laïcs, Gérard Altuzarra, directeur des ressources humaines, Philippe Leroux, informaticien analyste-programmeur, Pierre Adias, responsablecommunication, et d'un prêtre, le P. Régis-Marie de La Teyssonnière, ces deux derniers étant chargés de la rédaction.

Le projet obtient ensuite l'appui de l'évêque de Tarbes et Lourdes, Jacques Perrier, arrivé dans le diocèse en 1997. Homme d'expérience, puisqu'il fut évêque de Chartres, il doit préparer les jubilés de l'an 2000 pour l'Église catholique et de 2008 pour Lourdes $\left(150^{\mathrm{e}}\right.$ anniversaire des apparitions). De culture classique, brillant et travailleur selon les témoins rencontrés, il prend conseil auprès de laïcs, interroge les autres évêques, suscite le débat, puis réalise seul la synthèse. C'est aussi un homme de média : il est l'un des fondateurs de Radio Notre-Dame. Très à l'aise devant le micro ou face à la caméra, souriant, il sait vulgariser sans dénaturer le propos. Les journalistes l'apprécient, car il ne pratique pas la langue de bois. Soucieux de servir l'Église catholique, il réfléchit à l'utilité d'un sanctuaire comme Lourdes pour le $X X l^{\mathrm{e}}$ siècle. Monseigneur Perrier a l'intuition qu'une communauté de

\footnotetext{
${ }^{8}$ Entretien avec P. Adias, le 23 janvier 2014. En 1996, P. Adias est responsable de la communication; nous le remercions de son témoignage, et des documents qu'il a bien voulu nous remettre.
} 
Lourdes existe partout dans le monde de par le patronage de Notre-Dame de Lourdes sur des paroisses, des églises, des sanctuaires. II envoie le P. de La Teyssonnière faire le tour du monde pour les recenser et pour resserrer les liens avec le sanctuaire-mère. II faut établir des contacts avec tous ceux qui sont déjà venus à Lourdes, et avec tous ceux qui n'y viendront jamais : internet et le multimédia sont les moyens les plus pratiques et les moins chers, car il est hors de question, dans la perspective du jubilé de 2008, d'entreprendre de grands travaux ou de susciter des œuvres intellectuelles, comme a pu le faire Mgr Théas en vue du centenaire des apparitions en 1958 (Guinle-Lorinet, 1993). Mgr Perrier confie donc à des professionnels le soin de développer et d'enrichir le site ; il accepte ce que lui propose Mathias Terrier, entouré de toute une équipe de spécialistes en vue de la constitution d'un projet mix-media.

L'évêque actuel, Nicolas Brouwet, ancien auxiliaire de Nanterre, nommé le 11 février 2012, poursuit l'œuvre de son prédécesseur. Ses charges au sein de l'Église catholique de France, où il préside depuis avril 2011 le groupe de travail "Église et internet » l'y prédisposent. II souhaiterait le développement de l'outil par l'apport de nouvelles langues (chinois, arabe), de manière à toucher des communautés nombreuses ou celles de l'Église en détresse, qui représente les chrétiens en difficulté dans le monde. De plus, dans un livret sur l'évangélisation destiné aux fidèles du diocèse, il se fonde sur le chapitre 37 de l'encyclique de Jean-Paul II Redemptoris missio abordant l'intégration du message chrétien dans la nouvelle culture issue des communications modernes (Brouwet, 2014, p.75).

\subsection{Vers le dispositif actuel}

Le premier site internet a été présenté le 8 février 1996, aux directeurs de pèlerinages ${ }^{9}$, ce qui en fait un des premiers de l'Église catholique de France. II comprend deux parties : l'une concerne la ville ; l'autre est consacrée au sanctuaire. Ce site internet sera en constante évolution pour l'ergonomie bien sûr,

${ }^{9}$ Sanctuaire Notre-Dame de Lourdes (SNDL), « Un autre regard sur Lourdes », juin 2000, $12 \mathrm{p}$.

tic\&société - 9 (1-2), 2015 
mais aussi pour son contenu (ex. : ajout de la présentation du " domaine » du sanctuaire) et son périmètre. Certains services, celui chargé du patrimoine ou la revue Lourdes magazine ont eu des sites indépendants. En 1998, le site devient disponible en anglais; il le sera en espagnol en 2000. Cette année-là, l'évolution technologique permet désormais de déposer des intentions de prière et de diffuser le chapelet ${ }^{10}$ en direct, grâce à Radio Présence. Le 20 septembre 1998 est enregistré le millionième accès. Le site est en lien avec celui de la Conférence des évêques de France, dans la perspective de l'année jubilaire 2000.

C'est justement en 2000 qu'une étape capitale est franchie : une caméra est mise en place face à la grotte, sur la rive droite du Gave de Pau. D'une technologie semblable à celle des caméras de surveillance, cette webcam peut transmettre, quelles que soient les conditions météorologiques, des images sous plusieurs angles, selon plusieurs niveaux de zoom. Son emplacement permet de filmer la grotte, cœur du sanctuaire, et la procession quotidienne sur la prairie. Cinq programmes informatiques pilotent cette caméra ${ }^{11}$ : grotte, messes, chapelet, procession, nuit.

« Dès la mise en place de cette caméra, le 20 juin 2000, nous avons constaté une propagation très rapide de l'événement sur internet. En quelques jours le nombre quotidien d'accès au site internet de Lourdes a doublé », se félicitent les responsables $^{12}$. "Depuis cette date, les internautes du monde entier peuvent voir la grotte de Massabielle en vidéo, presque en direct (avec un décalage de quelques minutes) ainsi que les messes et autres événements qui s'y déroulent ${ }^{13}$. Devant un tel succès, une autre webcam est installée, le 11 octobre 2001, devant la basilique Notre-Dame du Rosaire ; elle capte de

${ }^{10}$ Dire le chapelet consiste à en faire passer tous ses grains entre les doigts en récitant, de façon alternée, notamment le «Je vous salue Marie... » et le «Notre Père », et en procédant à une visualisation mentale du "Mystère chrétien », étape par étape, à travers la figure de la Vierge Marie.

${ }_{11}^{11}$ Cette caméra a été changée en 2006. Communiqué de presse, n³7, 9 août 2006.

12 SDNL, « Mieux accueillir sur internet », conférence de presse, 15 mars 2004.

13 Idem. 
nouvelles vues des sanctuaires et permet aux internautes de suivre la procession nocturne aux flambeaux ${ }^{14}$.

Au début de l'année 2009, dernière étape, le sanctuaire se dote d'un service multimédia ${ }^{15}$, proposé dès 2008. Les TNIC, selon un concept de mix-media, servent à collecter, traiter de l'image et du son en un seul processus et à les diffuser en limitant les coûts sur la plupart des supports existants, Web-TV et web-radio, TV et radio hertziennes, réseaux sociaux, imprimés. Des stratégies de cross-media sont appliquées pour produire et diffuser les événements médiatiques. Pour la collecte des informations et des images, la grotte dispose de deux caméras TV fixes pilotables à distance. Celle qui filme la grotte presque de face à 40 mètres environ est utilisée la majorité du temps ; elle fut la première installée, et a plusieurs fois évolué afin de transmettre la meilleure qualité d'image possible. L'angle de prise de vue place le spectateur dans un axe de vision de la grotte proche de celui retenu pour Bernadette, la voyante, lors de la première apparition. ${ }^{16}$ Cet angle est le même que celui privilégié par les pèlerins sur place $^{17}$. Une deuxième caméra est située à l'entrée de la grotte pour filmer les pèlerins et les chapelains ${ }^{18}$ de face; elle est utilisée pour la retransmission de la messe ou du chapelet pour montrer l'assemblée ou le célébrant. Une des premières webcams mises en place, du type des outils de surveillance, subsiste, filmant l'esplanade du Rosaire et donc l'arrivée des deux processions quotidiennes, celle du Saint-Sacrement à $17 \mathrm{~h}$ et celle aux flambeaux à $21 \mathrm{~h}$. Des caméras sont aussi installées le long du parcours des processions, ainsi que dans la basilique Saint-Pie $X$ pour la retransmission des messes internationales avec écrans et vidéoprojecteurs sur place. Ce dispositif permet d'accueillir le final de la procession du SaintSacrement en épargnant l'attente au soleil, ou sous la pluie, aux malades. Ceci signifie que les images servent sur place

${ }^{14}$ Idem

${ }^{15}$ SNDL, «Lourdes, 6 millions de pèlerins et 4, 2 millions d'internautes. Pour eux, un nouveau site internet ", conférence de presse, 4 décembre 2009.

${ }^{16}$ Sur le sol, devant la grotte, une plaque témoigne de la place où priait Bernadette Soubirous le 11 février 1858, lors de la première apparition.

17 La caméra «principale» et son axe traduisent un souhait de considérer l'internaute comme un pèlerin au sanctuaire, de lui montrer ce qu'il verrait sur place (Terrenoire, 2006, p.128-131).

${ }_{18}$ Prêtres desservant le sanctuaire.

tic\&société - 9 (1-2), 2015 
également, et se mêlent au vécu du pèlerinage en présentiel, renforçant pour ces pèlerins le vécu de l'événement. Par ailleurs, un journaliste et un reporter photographe employés du sanctuaire réalisent des films ou des clichés à thème. Les prises de son se font à la grotte ou auprès des chorales. L'ensemble de ces outils sont interconnectés par un réseau souterrain de fibre optique à trois régies situées sous les arcades, dans la basilique souterraine, dans les locaux du pôle de communication. Un système de paraboles et de fibre optique assure l'envoi et la réception des contenus dans le monde entier.

Le dispositif a été conçu d'abord pour permettre aux pèlerins sur place de participer, puis pour proposer une image "brute » et permanente de la grotte. Devant les possibilités offertes par cet équipement, des télévisions comme KTO en France ou Canale 28 et TV 2000 en Italie ont demandé des émissions quotidiennes (récitation du chapelet, Angelus) qui atteignent des audiences moyennes parfois surprenantes (800 000 par jour pour le chapelet sur TV 2000 avec des pointes à un million) ${ }^{19}$. D'autres accords sont passés ou en cours de négociation, ils peuvent être ponctuels, pour des événements de Lourdes, ou réguliers, avec le Brésil, les États-Unis.

Les internautes disposent de nombreux outils. Un site en six langues totalise 9,1 millions de pages vues et 3,1 M d'entrées en $2013^{20}$ pour des visiteurs de plus de 150 pays. II permet de déposer en ligne des dons, des intentions de prière, des offrandes pour faire brûler un cierge (200 par jour) ; il fournit des textes de prières, des informations sur le fait de Lourdes (apparitions, miracles) et sur l'organisation des pèlerinages. Une WebTV retransmet en permanence la grotte ou les célébrations $(13,56 \mathrm{M}$ de pages vues, $8,5 \mathrm{M}$ de visites, visiteurs de plus de 140 pays), elle peut être considérée comme la « vraie » page d'accueil des outils internet. À partir d'elle, on accède à une chaîne sur Youtube disposant en 2013 de 560 vidéos (200 nouvelles/an) et totalisant 6,45 M de vues. Une page Facebook rassemble plus de 130000 fans de 45 pays. Avec ses reprises, l'image-message de la nouvelle année a été vue par 350000 personnes. Un compte Twitter informe principalement les professionnels ; il a plus de 2800 abonnés.

\footnotetext{
${ }^{19}$ KTO ne disposerait pas de chiffres d'audience pour les diffusions télévisées, en revanche sur le site internet de la chaîne, le programme du chapelet de Lourdes en direct et en différé est très suivi.

${ }^{20}$ Entretien avec L. Jarneau, le 13 février 2014.
} 
Enfin, une galerie de photos sur FlickR dispose de plus de 2400 images.

\subsection{Des buts pratiques, un enjeu pastoral}

Les TNIC remplissent pour Lourdes des buts pratiques. II s'agit de permettre aux internautes du monde entier de connaître l'histoire de Lourdes et des événements qui s'y sont déroulés, de trouver des renseignements sur le sanctuaire luimême, par exemple sur son patrimoine, d'obtenir des informations sur les pèlerinages, les retraites, les colloques qui s'y tiennent. Mais l'internaute peut aussi faire des dons en ligne, demander à ce qu'on brûle un cierge, déposer des intentions de prière, comme s'il était présent sur les lieux. II peut assister à la récitation du chapelet à la grotte, ou à des messes, ou encore suivre des processions. II peut aussi se recueillir seul devant la grotte, quand il ne s'y passe rien, la nuit par exemple.

Lourdes a toujours mis à son service les moyens modernes de communication. Par exemple la photographie, dont la Lourdes des apparitions est contemporaine : l'image de la grotte est ainsi fixée (Guinle-Lorinet et Bernadou, 2013), avant même que Mgr Laurence ne reconnaisse en 1862 la réalité des apparitions ; Bernadette Soubirous fut la première future sainte photographiée (Laurentin, 1978). La carte postale permit de faire connaître le sanctuaire en France et ailleurs. Lourdes, dès 1858, a fait la une de la presse locale, nationale, internationale ; et le sanctuaire très vite a su créer sa propre presse et structurer, contrôler ses relations avec les journalistes, grâce au bureau de presse, puis au service communication (Cailleaux, 2007). En 1987, la radio s'est installée dans le sanctuaire ${ }^{21}$, et c'est elle qui a permis les premières retransmissions du " chapelet ». Internet et le multimédia constituent la dernière étape de ce chemin médiatique. Tous ces moyens de communication ont été mis au service, chronologiquement et souvent simultanément, de la diffusion du "message » de Lourdes. Nous verrons que nous sommes cependant dans un modèle de communication davantage télégraphique

${ }^{21}$ La radio est fondée en 1987 sous le nom Radio Lourdes Bigorre, en 1993 elle obtient de nouvelles fréquences, sous le nom de Radio Lourdes Pyrénées; en 1999, elle établit des relations avec les radios régionales chrétiennes et devient Présence Lourdes Pyrénées.

tic\&société - 9 (1-2), 2015 
qu'orchestral qui peut se rencontrer dans d'autres expériences catholiques du web (Catellani, 2014, p.110).

Tout en remplissant des buts pratiques, les TNIC répondent aussi et surtout à des enjeux pastoraux, et parfois les suscitent. C'est un moyen technique pour diffuser ce que l'Église catholique appelle le "message de Lourdes », qui aurait été délivré par la Vierge Marie à Bernadette Soubirous. La transmission de ce " message » est à la charge du magistère, donc de l'évêque du lieu, dans le cadre du sanctuaire et en dehors. En 1958, les travaux historiques et théologiques de René Laurentin, commandés par l'évêque de Tarbes et Lourdes de l'époque, Mgr Théas, permirent de mieux définir ce message (Guinle-Lorinet, 1993). Il tient en quelques mots : pauvreté, prière, pénitence. Délivré dans un environnement naturel, il met en valeur l'eau, le rocher, la lumière, qui (re)deviennent des signes. Transmis par une jeune fille pauvre et souffrante, ce message s'adresse particulièrement aux malades. C'est ce message que viennent chercher à Lourdes les pèlerins et que caméra et TNIC s'efforcent de rendre visible sur les réseaux numériques. Lourdes, comme la plupart des lieux de pèlerinage, constitue, pour l'Église catholique, un instrument privilégié de communication de la foi car il rassemble une communauté protéiforme bien au-delà des fidèles pratiquants (Bernard, 2013). De plus, il s'est révélé particulièrement assimilable par les habitudes de la société de consommation qui en amplifie la diffusion (Kaufman, 2005).

\section{Un questionnement par l'anthropologie}

L'approche des faits communicationnels religieux par l'anthropologie que nous proposons consiste à les étudier d'emblée comme des faits de communication interculturelle. Nous distinguons grâce au travail ethnographique ${ }^{22}$ les

${ }^{22}$ Le travail ethnographique a duré plus de six années, il a comporté deux phases, une ethnographie du sanctuaire de Lourdes et une étude des médiatisations de ce fait religieux. La posture épistémologique adoptée était l'ethnométhodologie.

Pour l'ethnographie dans le sanctuaire, nous avons adopté plusieurs « rôles » qui ont conditionné fortement les interactions: pèlerin "isolé ", accompagnateur d'un pèlerinage diocésain, d'un pèlerinage Lourdes cancer espérance (LCE), participation en tant que malade et accompagnateur au pèlerinage Rénalis (malades des reins). Ces expériences ont été recueillies sur 3000 pages de cahier de terrain et 400 photographies réalisées. II faut ajouter des témoignages : le recueil des échanges de 
composantes majeures du fait communicationnel, pour Lourdes le fait religieux catholique et la modernité. Chaque composante possède des traits spécifiques, parmi lesquels on peut citer des personnages (par ex. les prêtres, les fidèles, les pèlerins... pour la tradition catholique ; les scientifiques, les médecins, les journalistes..., pour la modernité), des pratiques sociales spécifiques (par ex. la participation aux offices religieux pour la tradition catholique, la production et la consultation des journaux d'information imprimés, radiophoniques ou télévisés pour la modernité) mais aussi un certain rapport au temps (par ex. plutôt cyclique, avec la prégnance du calendrier liturgique et de ses fêtes pour le fait religieux; plutôt dans l'instant ou l'évènement ponctuel pour la modernité, comme le constate Régis Debray au sujet de la vidéosphère). Lorsque le travail de repérage des traits spécifiques est mené, avec des attributions au fait religieux ou à la modernité, quand cela est possible, nous pouvons regarder le fait de Lourdes dans son ensemble et identifier les dynamiques qui le traversent, venant de la tradition religieuse ou de la modernité, et constater si ces dynamiques se heurtent ou se complètent, ou bien les deux à la fois. L'idée

pèlerins lors des carrefours de LCE (1200 pers./an), sur deux ans, des entretiens individuels approfondis avec des personnes références (participants et organisateurs de pèlerinage, cancérologue, théologien, une personne athée et une agnostique). Pour l'étude des médiatisations, nous avons réalisé trois collections : d'images de Lourdes diffusées, d'articles sur Lourdes de quotidiens nationaux, de corpus sur Lourdes réalisés par bibliothèques de référence nationales, scientifiques, religieuses. La collection d'images a permis, à partir d'une consultation de 30000 images d'en analyser 207 de manière méthodique, classées par thèmes et périodes sur 150 ans, afin de comprendre les valeurs communiquées. Les articles de journaux ont été repérés de manière systématique sur quatre périodes espacées de 50 ans, pour chaque période quatre titres ont été retenus, censés couvrir les principales opinions de la société française sur Lourdes. Le contenu de chaque article a été analysé de manière quantitative et qualitative et mis en relation avec le tirage des quotidiens pour tenter d'en inférer la force des différentes opinions sur Lourdes dans la société française et leur évolution dans le temps. À partir de l'interrogation des catalogues de bibliothèques de référence nous avons établis les corpus de documents que chacune possédait sur Lourdes. Cela concerne les bibliothèques nationales d'Algérie, d'Afrique du Sud, d'Australie, de Belgique, du Brésil, des États-Unis, de France, d'Israël, d'Italie, de Turquie, du Royaume-Uni, les bibliothèques scientifiques de la bibliothèque nationale de médecine des États-Unis (Medline) et la bibliothèque InterUniversitaire-Médicale de Paris, les bibliothèques religieuses de Dayton, Lourdes, Louvain, Rome (Marianum) et du Vatican. L'analyse quantitative et qualitative des corpus d'ouvrages sur Lourdes de chaque bibliothèque permet en particulier pour les nationales de comprendre à travers quels courants d'idées (religieuses, médicales...) Lourdes se communique ou pas. Ceci esquisse des canaux de la communication interculturelle de Lourdes.

tic\&société - 9 (1-2), 2015 
ici est de reprendre les schismogenèses ${ }^{23}$ symétriques et complémentaires (Hennin, 1993) - heurt ou complémentarité de Gregory Bateson (1977, pp.265-266), ou bien un mélange des deux, phénomène repéré en biologie, appelé agoantagonisme (Bernard-Weil, 2002). Par exemple, lors de la fête de Notre-Dame de Lourdes, le 11 février, on constate un accroissement de trafic sur la webcam, c'est alors clairement le fait religieux qui en est à l'origine. II est intéressant d'analyser les publics qui se connectent grâce aux données fournies par les TNIC. Lorsque le sanctuaire de Lourdes subit des inondations, les connections augmentent aussi ; nous sommes alors davantage dans de l'évènementiel, au sens de la modernité. Mais comment ces deux types d'évènements entrent-ils en interaction ? Un accroissement du trafic pour la fête de Notre-Dame de Lourdes va-t-il influencer le nombre de connexions en cas d'intempéries ? Et ce, à la hausse ou à la baisse? Les inondations dans le sanctuaire visibles par la webcam vont-elles inciter les pèlerins à venir à Lourdes ou les en dissuader? Les inviter à se connecter, ou non, à la webcam, lors des futures fêtes liturgiques ? Quels sont les enjeux ? Comment les équipes du sanctuaire les gèrent-ils ? Telles sont les questions problématisées grâce au concept de schismogenèse de Bateson ou à l'ago-antagonisme. Ce travail de déconstruction, repérage et reconstruction aide à penser l'espace de signification socialement partagé et à en inférer les comportements et attentes des utilisateurs des TNIC mises à disposition par le sanctuaire.

\subsection{Quelques notions sur le pèlerinage}

L'article d'Alphonse Dupront "Pèlerinages et lieux sacrés » (1983) donne une définition transculturelle de l'anthropologie du pèlerinage pour poser les principales notions utilisées et leurs rapports; certains aspects se trouvent complétés par les travaux de Victor et Édith Turner (1978). Trois notions

23 Schismogenèse : processus de différenciation des normes de comportement individuel résultant d'interactions cumulatives entre les individus ; schismogenèse symétrique : schismogenèse dans laquelle les parties qui se stimulent mutuellement produisent des actions similaires (compétition, rivalités); schismogenèse complémentaire: schismogenèse dans laquelle les parties qui se stimulent mutuellement produisent des actions dissemblables mais réciproquement appropriées (domination et soumission, assistance et dépendance). 
apparaissent indissociables: lieu sacré, quête humaine du sacral, volonté de puissance collective et individuelle.

Les lieux sacrés sont, selon Dupront, comme émergences nécessaires, dans l'indéfini d'un espace physique homogène. Ils sont d' " une nature différente du milieu environnant, hétérogènes à celui-ci, caractérisés comme autant de points d'un ailleurs déterminé, qui orientent et fixent la recherche collective et individuelle d'un état "autre" " (Dupront, 1983, p.804). Ils sont pour les pèlerins un lieu géographique qui a une transposition psychique, lieu-source, souvent au sens concret, et de ressourcement. Le pèlerinage représente "la geste extraordinaire d'une quête humaine du sacral [...] entreprise dans la libre disposition de soi.» (Ibid.), c'est un acte d'offrande. II est « épreuve de la force personnelle de croire et il porte témoignage de la vérité sacrale du lieu où il s'accomplit.» (Ibid.) II est volonté de puissance collective ou individuelle d'exister en un corps humain qui parcourt un chemin, puissance de disposer de sa vie (Ibid.).

Lourdes est un pèlerinage particulier; la volonté de puissance collective ou individuelle d'exister en un corps humain ne s'éprouve pas tant dans un chemin parcouru, même s'il est bien réel, que dans le " prendre soin " ensemble des personnes malades, handicapées, qui sont parfois en attente de guérison. D'autres actes spécifiques correspondent aux paroles de l'apparition et sont liés au lieu : boire à la source et s'y laver, venir en procession.

Pour Alphonse Dupront et Victor Turner, le pèlerinage " apparaît comme l'une des formes les plus totales de l'accomplissement des rites de passage» (Dupront, 1983). Le pèlerinage produit " une société fraternelle de salut commun», éphémère et indifférenciée que Turner appelle communitas (Turner, 1969, p.97-98). II apparaît comme un des espacestemps de liminarité ${ }^{24}$, comme un lieu de potentialités où se formulent les transformations des structures sociales (Turner et Turner, 1978) ou des réactions aux changements de contextes sociaux.

\footnotetext{
${ }^{24}$ La notion de liminarité ou liminalité réfère aux travaux de van Gennep sur les rites de passage, repris par $\mathrm{V}$. Turner. Ces rites marquent le changement de statut social ; ils comportent trois étapes : la séparation (l'individu est isolé du groupe), la liminarité (il demeure à la marge du groupe), la ré-agrégation (il réintègre le groupe avec un nouveau statut).
}

tic\&société - 9 (1-2), 2015 
Pour les pèlerinages chrétiens nés au $\mathrm{XIX}^{\mathrm{e}}$ siècle, la piété personnelle est mise en avant; ils se développent grâce aux moyens de communication et aux technologies de l'époque moderne dont ils dépendent (trains, journaux, éditions de livres, d'images de piété et de cartes postales...). Ils forment un " système apologétique » chrétien déployé contre l'avancée de la sécularisation dans la société de masse, urbanisée et influencée par l'épistémologie issue du darwinisme ${ }^{25}$ qui pose problème à la cosmo-anthropologie ${ }^{26}$ traditionnelle chrétienne, signes d'une adaptation au conflit d'épistémologie avec le positivisme au sens large, comprenant la théorie de l'évolution.

\subsection{Pèlerinage et " webpèlerinage »}

Les religions existant en bonne part via l'espace de signification socialement partagé qu'elles diffusent, le pèlerinage "physique " à Lourdes et la visite des sites du sanctuaire à l'aide des TNIC participent-ils ou non d'un même espace de signification socialement partagé ? Parler d'un webpèlerinage n'opère-t-il pas une annexion par l'espace de signification du pèlerinage, des utilisateurs des dispositifs de TNIC mis à disposition par le sanctuaire ? II serait tentant de trouver un "relais de croissance " via les TNIC alors que les pèlerins au sanctuaire de Lourdes, à très forte majorité européenne, sont touchés par la crise économique et l'érosion de la pratique religieuse traditionnelle. Mais si l'on se reporte aux caractères qui définissent un pèlerinage - nécessité d'une rupture avec le quotidien, épreuve de soi dans le parcours d'un

${ }^{25}$ Les apparitions de Lourdes à l'origine du pèlerinage précèdent d'une année la publication de L'Origine des espèces (1859) de Ch. Darwin, mais les questions sur l'évolution avaient émergé au moins un demi-siècle avant.

26 Nous employons ce terme de cosmo-anthropologie pour faire ressortir les spécificités liées aux conceptions du cosmos et de l'Homme. Selon l'approche en sciences humaines et sociales issue de la modernité que nous développons la conception anthropologique inclut implicitement la conception cosmologique. Avec I'Incarnation sous-jacente au fait religieux chrétien que nous étudions, qui récapitule la création dans la personne du Christ, il en est de même mais sous une forme très différente. Ainsi par habitude on en vient à oublier que la différence de conception entre modernité et beaucoup de religions va au-delà de la conception de l'Homme (lié ou non à un dieu) et s'étend au cosmos, à nos manières de le concevoir et de le penser et d'entrer en interaction avec lui, comme le montre Bruno Latour (1997). En d'autres termes, l'espace de signification représenté par le cosmos n'est pas socialement partagé, l'emploi du terme cosmo-anthropologie vise à permettre de le souligner. 
chemin vers ou sur un lieu sacré, production d'un espacetemps de liminarité et d'une communitas - il est moins évident de parler de web-pèlerinage, car les études sur les utilisateurs et sur leurs comportements apparaissant maintenant comme nécessaires n'en sont encore qu'à leurs balbutiements. Comment savoir dès lors qui ils sont? Quelles sont les valeurs qu'ils partagent et sont-elles les mêmes que celles du pèlerinage « physique » à Lourdes ${ }^{27}$ ?

En l'absence de données d'étude permettant de définir directement les espaces de signification socialement partagés ou non entre pèlerins de Lourdes et utilisateurs des TNIC, il conviendrait de remonter d'une étape logique pour fournir quelques éléments concrets de réponse. Un tel espace est soutenu par une "cosmo-anthropologie " ou des "cosmoanthropologies » qui se recoupent; les "cosmoanthropologies " produites par les pèlerins et par les internautes présentent-elles des traits descriptifs similaires ou différents ou bien ces traits pourraient-ils s'intégrer dans une " cosmo-anthropologie » de Lourdes d'avant l'organisation des pèlerinages ? Pour répondre à ces questions, il faut repérer succinctement certains de leurs caractères simples : la place de la grotte, les personnages spirituels et physiques, les services des sanctuaires impliqués, les messages religieux et profanes véhiculés, les publics concernés, les actes posés, les temporalités, les flux financiers. Dans la mesure du possible, des logiques d'interprétation des comportements reliant les faits seront signalées. Les TNIC, grâce au suivi des connexions, fournissent à ce niveau des données pertinentes.

\subsubsection{Des traits similaires...}

La prépondérance des connexions à TV Lourdes, centrée sur la grotte avec 8,5 M de visites en 2013 (contre 3,1 M pour le site d'information lourdes-france.org) affirme une focalisation

\footnotetext{
27 Répondre à ces questions orientait la recherche vers l'emploi de démarches qualitatives pour connaître le comportement des internautes. Ces démarches, impliquant un certain coût de mise en œuvre, n'ont pas été au moment où nous écrivons réalisées par le sanctuaire. C'est là que l'apport des TNIC s'est manifesté ; en effet nous avons constaté que le foisonnement des données quantitatives permettait, grâce à une connaissance du terrain longue de plusieurs années, en dépassant le risque de l'entropie et à moindre coût, de faire ressortir des informations habituellement recueillies par voie qualitative.
}

tic\&société - 9 (1-2), 2015 
des internautes comme des pèlerins physiques sur la grotte, cœur du sanctuaire depuis le temps initial des apparitions. Parmi les pages du site lourdes-france.org, celles du dépôt d'intentions de prières à la grotte en français, italien, anglais et espagnol sont les quatre pages les plus utilisées (plus $1 \mathrm{M}$ de fois). La prière à la grotte reste clairement centrale (plus de $15 \%$ des consultations concernent ces quatre pages sur plusieurs milliers). Les pages permettant les dépôts de cierges en ligne représentent $3,5 \%$ des consultations pour celles en italien, français, anglais. Comme le déclare $M$. Terrier en entretien, à ce niveau les TNIC n'ont pas « révolutionné la démarche de prière ". Les pages sur l'organisation des pèlerinages physiques (calendrier, horaires, séjours à Lourdes, approfondissement des actes et de l'histoire du pèlerinage) suivent au nombre des connexions. La prépondérance des connexions concernant les dépôts d'intentions de prière puis les cierges correspond à la mise en œuvre de logiques dévotionnelles qui attestent fort probablement de la présence dans les " cosmo-anthropologies » des internautes des figures spirituelles de Lourdes : la Vierge, Bernadette. Pour la présence de Jésus, et du Dieu trinitaire des chrétiens, il faut être prudent $^{28}$. Notons qu'en nombre de connexions, l'activité dévotionnelle en ligne, intentions de prières et cierges, dépasse la recherche d'aide à l'organisation du pèlerinage physique. En outre, le comportement des pasteurs ou des hospitaliers (bénévoles qui aident dans le sanctuaire) se trouve modifié par les TNIC. Ceci conforte l'idée d'un espace de signification qui suit un processus d'unification entre pèlerins "physiques » et utilisateurs des TNIC. Souvent par des expériences en rapport avec leur sphère de connaissances personnelles, les pasteurs et l'évêque en tête prennent conscience que leurs célébrations sont suivies via les TNIC par une communauté invisible mais qui donne des signes de sa présence ${ }^{29}$. Ceci les conduit à modifier leurs comportements, l'évêque dira un mot d'ouverture en plusieurs langues même lors de la messe suivie dans le froid

${ }^{28}$ La perception de Lourdes en tant que lieu saint fait que le sanctuaire est fréquenté par des adeptes d'autres religions que les confessions chrétiennes; pourquoi la WebTV du sanctuaire ne le serait-elle pas aussi ? Par ailleurs les chapelains ont toujours eu le souci de combattre la mariolâtrie en ancrant les dévotions à la Vierge dans la christologie, par exemple par la procession du Saint-Sacrement.

${ }_{29}$ L'évêque peut recevoir un SMS de remerciement de l'étranger alors qu'il a célébré au petit matin à la grotte devant une assistance très réduite; tel prêtre sait que sa famille à l'autre bout du monde le verra célébrer ou bien une internaute coréenne demande par e-mail pourquoi la grotte est gardée, car elle a pris les hospitaliers pour des vigiles. 
de la nuit du $1^{\text {er }}$ janvier par une assistance très locale; les pasteurs saluent la communauté présente invisiblement par les TNIC, les hospitaliers adoptent des attitudes moins " martiales » alors qu'ils régulent le passage à la grotte.

Si nous examinons les temporalités, le logiciel Google analytics qui permet une analyse fine des consultations des sites internet montre que leur nombre est fortement influencé par le temps liturgique de Lourdes, tout comme la fréquentation du sanctuaire. Alors que le nombre moyen de consultations quotidiennes de TV Lourdes est de 23000 en 2013, il passe à 84000 le 11 février et le phénomène se reproduit chaque année. Sur le plan financier, les TNIC qui ouvrent vers l'extérieur procurent un surcroît de recettes clairement identifiable mais marginal, en temps normal, par rapport au pèlerinage et aux dons habituels: le dépôt de cierges en ligne apporte des recettes de $270500 €$ sur un budget de 30 M€ pour l'ensemble du sanctuaire soit moins de $1 \%$. Les dons en ligne, en période normale, représentent seulement $2 \%$ des collectes. En contrepartie, les investissements spécifiques pour les TNIC ouvrant sur l'extérieur sont contenus, car une bonne partie est mutualisée avec les fonctionnements internes du sanctuaire, par exemple l'équipement spécifique nécessaire pour la retransmission du chapelet pour TV2000 est de $50000 €$.

La convergence de ces traits descriptifs corrobore l'hypothèse d'une extension de l'espace de signification socialement partagé du pèlerinage à Lourdes aux utilisateurs des TNIC mises à disposition par le sanctuaire, mais certaines données poussent à aller plus loin dans la recherche. Par exemple, les membres du service communication incitent les pèlerinages organisés à bien préparer leur passage à la grotte qui est filmée quasiment en permanence ; ils ont observé des changements de comportements des pèlerins, la fascination de l'image opère, certains envoient un bonjour à la caméra, d'autres vont jusqu'à donner rendez-vous. Percevant cela, la chaine TV2000 a mis en place un dispositif en libre accès permettant d'envoyer des vidéo-messages depuis les sanctuaires - mais dans de tels cas, qui salue-t-on? des pèlerins qui n'ont pas pu se déplacer? ou est-ce un nouveau format de "carte postale de vacances " aux amis ou à la famille qui ne partagent absolument pas l'espace de signification religieux? 


\subsection{2. ... et des traits différents}

L'examen des différences de traits entre pèlerins à Lourdes et utilisateurs des TNIC peut aider à comprendre s'ils sont signes de logiques d'action différentes susceptibles de soutenir des espaces socialement partagés différents.

Les publics d'abord, même si en 2002 les pèlerins officiellement recensés venaient de 72 pays, le pèlerinage concerne essentiellement les pays européens (96\%, chiffre en lente diminution). Même si nous ne disposons pas de chiffres sur la même période, notons que sur 53705 visites sur le site d'informations lourdes-france.org issues de plus de 150 pays lors de l'anniversaire de la première apparition le 11 février 2014, les États-Unis, le Mexique, l'Argentine, le Brésil et le Canada faisaient partie des dix pays les plus connectés et représentaient $24,9 \%$ des visites ${ }^{30}$. La répartition des "fans " de Lourdes sur Facebook interroge aussi ${ }^{31}$. Le sanctuaire est fréquenté en majorité par des personnes de 55 à 75 ans, ce qui n'exclut pas pour autant des groupes d'adolescents et de jeunes. Il est donc surprenant de constater que la proportion de femmes des classes $25-34,35-44$ et $45-54$ ans montre une surpondération notable, $42 \%$ (pour ces trois classes) de tous les fans de Lourdes hommes compris. Cette surpondération est d'autant plus remarquable qu'elle est le double des deux classes 35-44 et 45-54 rapportées à l'ensemble des utilisatrices du réseau Facebook, or, ces classes sont très peu présentes physiquement dans le sanctuaire. Est-on en face d'un lien de substitution?

La grotte est le lieu de focalisation dans les deux cas mais la manière de l'aborder diffère par certains aspects. Dans le sanctuaire les pèlerins vont prier à la grotte mais ils passent aussi dans la grotte, pour cela ils font la queue parfois longuement, donnant forme à un corps social et prenant conscience de leur appartenance à cette communitas où le nombre estompe les différences. Selon leurs motions spirituelles, ils peuvent s'attarder devant la source qui coule ; ils touchent le rocher dur, poli, froid et humide. Ils ressentent la protection du froid et/ou de la chaleur que procure la grotte et voient la statue de la Vierge au sortir de cette expérience s'ils

\footnotetext{
${ }^{30}$ Données analysées en avril 2014.

31 Rappelons que le réseau Facebook met à disposition des détenteurs de pages une fonction permettant de rapprocher les classes d'âge des fans de la page avec celles de l'ensemble du réseau.
} 
se retournent. Ils peuvent aller boire l'eau qu'ils ressentent fraîche ou froide les pénétrer, les désaltérer, couler sur leurs membres endoloris ou malades. Ils sont confrontés à la dimension incarnée de leur croyance qui ré-ouvre souvent leur conscience à la dimension symbolique des actes qu'ils posent, qui deviennent signes et source d'une réinterprétation des événements de leur vie. Cela ouvre des espaces fermés par les pré-pensées efficaces de la vie quotidienne avec ses comportements dépersonnalisés et automatiques. Cette différence de logique, abordée au sujet de l'emploi du terme cosmo-anthropologie, est décrite par B. Latour dans son ouvrage Nous n'avons jamais été modernes. La grotte peut jouer un rôle de réouverture aux archétypes portés par la nature (Guinle-Lorinet et Bernadou, 2013), touchant en profondeur aux fonctions métacognitives des personnes (Melot, 1998). Là où les TNIC mobilisent la vue et l'ouïe, l'expérience du pèlerinage rajoute l'odorat, le toucher et le goût, ouvrant à une expérience de liminarité ou « re-matriciante » (la grotte figurant une matrice) objectivement plus profonde, ainsi qu'à la dimension symbolique de la vie humaine. Ce qui permet d'opérer des liens entre le monde sensible et le monde intelligible autrement que par abstraction comme le fait l'épistémologie positiviste (Bernadou, 2008). Un de nos interlocuteurs nous a clairement dit qu'un service d'envoi par internet d'eau de la grotte n'est pas développé car il est difficile de faire comprendre que l'eau n'est pas porteuse d'une vertu de guérison mais qu'elle est simplement le signe de la grâce. Pour l'épistémologie positiviste qui s'est développée dans le sillage de la modernité, un élément naturel ne réfère à rien d'autre que lui-même, ses propriétés physiques, ou ses références sociales. La logique du signe ici décrite s'en trouve supprimée. Cela va de pair avec la notion de sacrement qui est inaccessible à la pensée moderne, même si les sacrements restent distribués. Les sacrements de l'Église catholique ne sont pas accessibles par les TNIC.

La suppression de l'expérience de plusieurs sens peut amputer celle-ci et la faire entrer dans un autre type de logique qui gomme la dimension temporelle et les processus au profit de l'instant. L'examen de la temporalité fournit aussi des différences de comportement entre le pèlerinage et l'utilisation des TNIC qui sont plus sensibles à la dimension événementielle profane. En ce sens, les événements climatiques comme les inondations représentent des pics d'audience sur les sites

tic\&société - 9 (1-2), 2015 
internet. En cas d'aléas climatiques, les comportements financiers et les flux s'en trouvent modifiés. Les dons spontanés se révèlent plus nombreux et de plus grande importance que les réponses aux sollicitations (51\%). La fréquentation des sites internet est telle, particulièrement en ces moments de forts besoins financiers, qu'il importe de réfléchir aux moyens de capter la générosité. La fonction de dons en ligne est « mieux repérée ${ }^{32}$ " par les utilisateurs et la proportion des dons en ligne augmente, passant de 2 à $7 \%$. Les dons par courrier, euxmêmes, sont souvent accompagnés de mots indiquant que les donateurs ont suivi les événements grâce aux TNIC, mais pas exclusivement celles proposées par le sanctuaire. Les effets mix-media et cross-media peuvent être ici convoqués quand le directeur de la chaine hertzienne TV2000, qui retransmet le chapelet de Lourdes, remet un chèque de $768000 €$, résultat d'une collecte auprès des téléspectateurs ou qu'une religieuse coréenne collecte $10000 €$ attestant que dans son diocèse les fidèles ont suivi les actualités par internet. Mais rien ne dit dans ces cas que nous sommes dans l'espace socialement partagé du pèlerinage.

\subsubsection{L'intégration à un espace socialement partagé "Lourdes » originel}

Avant d'être un lieu de pèlerinage impliquant une certaine régulation par l'institution religieuse, Lourdes a été un fait d'apparition avec ses traits spécifiques. Le développement de l'utilisation des TNIC mises à disposition par le sanctuaire ne porte-t-il pas les traits spécifiques du fait d'apparition originel ? Pour resituer la réflexion par rapport aux travaux d'Alphonse Dupront, la dimension du lieu sacré (d'une nature autre que l'espace environnant) prime sur celle du pèlerinage, surtout celle du pèlerinage à Lourdes qui est très encadré par l'Église catholique.

Le fait d'apparition initial impliquait la grotte, Bernadette Soubirous, une apparition et des foules de toutes conditions qui se rassemblaient : personnes en prière, curieux, sceptiques et forces de l'ordre qui surveillaient de loin et faisaient l'inventaire des personnes présentes et des actes posés (dépôts de cierges, d'argent) en l'absence de représentants officiels de l'Église catholique. C'est l'apparition elle-même qui a

${ }^{32}$ Entretien avec T. Castillo, le 13 février 2014. 
« convoqué » l'Église, demandant à la voyante lors de la treizième apparition, le 2 mars 1858: «Allez dire aux prêtres qu'on vienne ici en procession et qu'on y bâtisse une chapelle ». La logique de l'apparition de Lourdes, qui pourrait être qualifiée de dogmatique avec le nom donné par l'apparition "Que soy era Immaculada concepciou», semblait venir confirmer de son côté plusieurs siècles de réflexion ecclésiale pour savoir comment Dieu pouvait s'incarner dans une lignée humaine touchée par le péché. C'était bien la logique et la possibilité d'un contact entre terre et ciel, divin et humain qui se jouait, alors que l'anthropologie de la connaissance issue de la modernité formalisée dans le positivisme conduisait par principe de méthode à l'évacuer.

Comme lors des apparitions, avant l'intervention de l'autorité ecclésiale, la foule vient à la grotte par les TNIC, c'est un lieu de dépôt des intentions de prière et de cierges, accessible facilement. Le chapelet est le mode de prière qui opère le mieux comme lors des apparitions. Il produit de la communauté ; par son aspect répétitif il demande un effort personnel et a des consonances dans la plupart des religions. Instinctivement, les pasteurs perçoivent que leur rôle est davantage d'accompagner le mouvement que de le diriger, peut-être est-ce pourquoi le service communication du sanctuaire est placé aux avant-postes? La propension unificatrice en apparence peu contraignante des TNIC semble s'accorder à la piété populaire qui nécessite parfois d'être canalisée. Victor et Édith Turner qui ont proposé l'idée de communitas ne renieraient pas le discours du directeur du pôle communication: " internet avec le multimédia est l'outil rêvé pour Lourdes, la communauté mondiale que constitue Lourdes avait besoin d'un outil qui puisse faire du lien " ${ }^{33} "$. L'économe, lui, constate les effets sur la structure interne de la communication du sanctuaire qui était très segmentée selon les différents média et qui se trouve maintenant unifiée. II a intégré dans ses budgets que la qualité d'image haute définition était maintenant la norme pour les équipements vidéo des sanctuaires, même à destination des pèlerins à Lourdes. La même logique est suivie pour la réalisation des messes internationales ou des processions; les plans larges sont intercalés entre les plans serrés afin que " tout le monde » entre dans la célébration. Pour reprendre le contexte de

${ }^{33}$ Entretien avec M. Terrier, le 13 janvier 2014.

tic\&société - 9 (1-2), 2015 
l'apparition, la foule, par les TNIC, est présente comme en 1858, la chapelle de pierre est construite, la chapelle virtuelle est en construction ; comment former les processions (virtuelles) ?

\section{Conclusion}

Au terme de notre parcours, en tant que chercheurs travaillant d'assez longue date sur le fait de Lourdes, nous avons perçu une homogénéité du développement des TNIC avec ce qui fait l'histoire de Lourdes, la focalisation sur la grotte, les figures de Bernadette et de la Vierge, la présence de la foule. La grotte tenant le rôle de l'Agora antique comme centre de l'espace public (Gadras et Paillart, 2013, p.24), les TNIC à Lourdes semblent consacrer son rôle central d' " incarnation » d'un " espace public " de prière (Idem, p.36). Lourdes a une tradition de précurseur ${ }^{34}$ pour l'Église catholique française en termes d'innovation technique pour la communication qui se vérifie avec l'utilisation des TNIC. Ce mode de développement n'empêche pas les tâtonnements de début comme pour les sites internet, puis viennent quelques idées structurantes, la webcam filmant la grotte en permanence, l'intégration dans des projets-cadres ; les TNIC ont suivi un processus habituel à Lourdes qui reflète la dynamique de ce fait religieux. Lourdes n'a pas suivi le développement des TNIC mais se développe en osmose avec elles : en 2012, 6,6 \% des internautes se connectaient à TV Lourdes via tablettes et smartphones, en 2013 ils atteignaient 15,2\%, Ipad et Iphone en tête. Le $150^{\circ}$ anniversaire des apparitions en 2008 n'aura pas produit de constructions matérielles déterminantes mais il aura scellé et ancré en profondeur l'ouverture du sanctuaire au « continent » internet.

L'emploi du terme webpèlerinage, même s'il n'est pas impropre à refléter certaines pratiques, semble prêter un peu à caution. On ne peut pas dire que le pèlerinage à Lourdes a son équivalent en version numérique. D'abord, le pèlerinage " physique » y est un modèle très spécifique de pèlerinage, il ne se passe pas tant sur le chemin, qui existe néanmoins, qu'en processions et soins aux personnes malades ou handicapées sur le lieu sacré du sanctuaire, aux alentours de la grotte. Certains actes posés par les utilisateurs des TNIC

${ }^{34}$ La décision de sonoriser l'ensemble du sanctuaire avait été prise dès l'année 1931. 
fournies par le sanctuaire sont authentiquement religieux et homogènes à ceux des pèlerins sur place. En ce cas, les TNIC fournissent un accompagnement info-communicationnel de la pratique qui aboutit à une extension de l'espace de signification socialement partagé du "fait religieux de Lourdes ». Ensuite, il nous semble que l'expression "fait religieux de Lourdes » correspond plus précisément que «pèlerinage " à l'élément central de ce qui se déploie via les TNIC ainsi qu'à l'état de formalisation des pratiques religieuses en cours. Enfin, les TNIC du sanctuaire ouvrent un accès qui peut se révéler précieux à un espace de signification socialement partagé d'ordre profane, très sensible aux faits divers, mais qui est fortement différencié de celui produit par le fait religieux.

La dernière remarque peut faire écho à la réflexion sur l'Incarnation comme auto-communication de Dieu (Douyère, 2013). Le fait de Lourdes a acquis sa dimension ecclésiale précisément le 25 mars 1858, date à laquelle Bernadette donne le nom de sa vision au curé Peyramale. C'est aussi le jour où l'Église catholique fête l'Incarnation; le nom qui a été inscrit, bien lisible par tous les pèlerins, au pied de la statue de la grotte, est: "Que soy era immaculada concepciou » (Je suis l'Immaculée conception), soit, compte tenu des mots et du contexte, celle en qui l'Incarnation est en train de se faire, passage de l'éternité au temps, en régime chrétien. L'attitude contemplative connue chez les pèlerins de Lourdes, qui, à la grotte, paraissent oublier le temps qui passe, semble étonnamment se retrouver chez certains internautes face aux images de la webcam de la grotte. Ceci pourrait expliquer la place de Lourdes dans la société mondialisée, grâce, en bonne partie, aux TNIC. Les sanctuaires d'Aparecida au Brésil ou de Guadalupe au Mexique affichent un plus grand nombre de pèlerins " physiques " que Lourdes, mais il est significatif que sur le continent " internet » où ces sanctuaires ont aussi pris pied et parfois avec des moyens matériels et humains plus importants que ceux de Lourdes ${ }^{35}$, ces faits restent à portée essentiellement nationale. Lourdes, comme en attestent les statistiques de connexion, dispose d'un potentiel de communication international et transculturel dont les autorités ecclésiastiques en charge du sanctuaire cherchent sans cesse, par l'intégration de nouvelles langues (chinois, arabe) et des TNIC, à déployer la communication et/ou l'Incarnation.

\footnotetext{
${ }^{35}$ TV Aparecida aurait plus d'une centaine de salariés.
}

tic\&société - 9 (1-2), 2015 
Une webcam à la grotte : Lourdes et l'introduction des TNIC

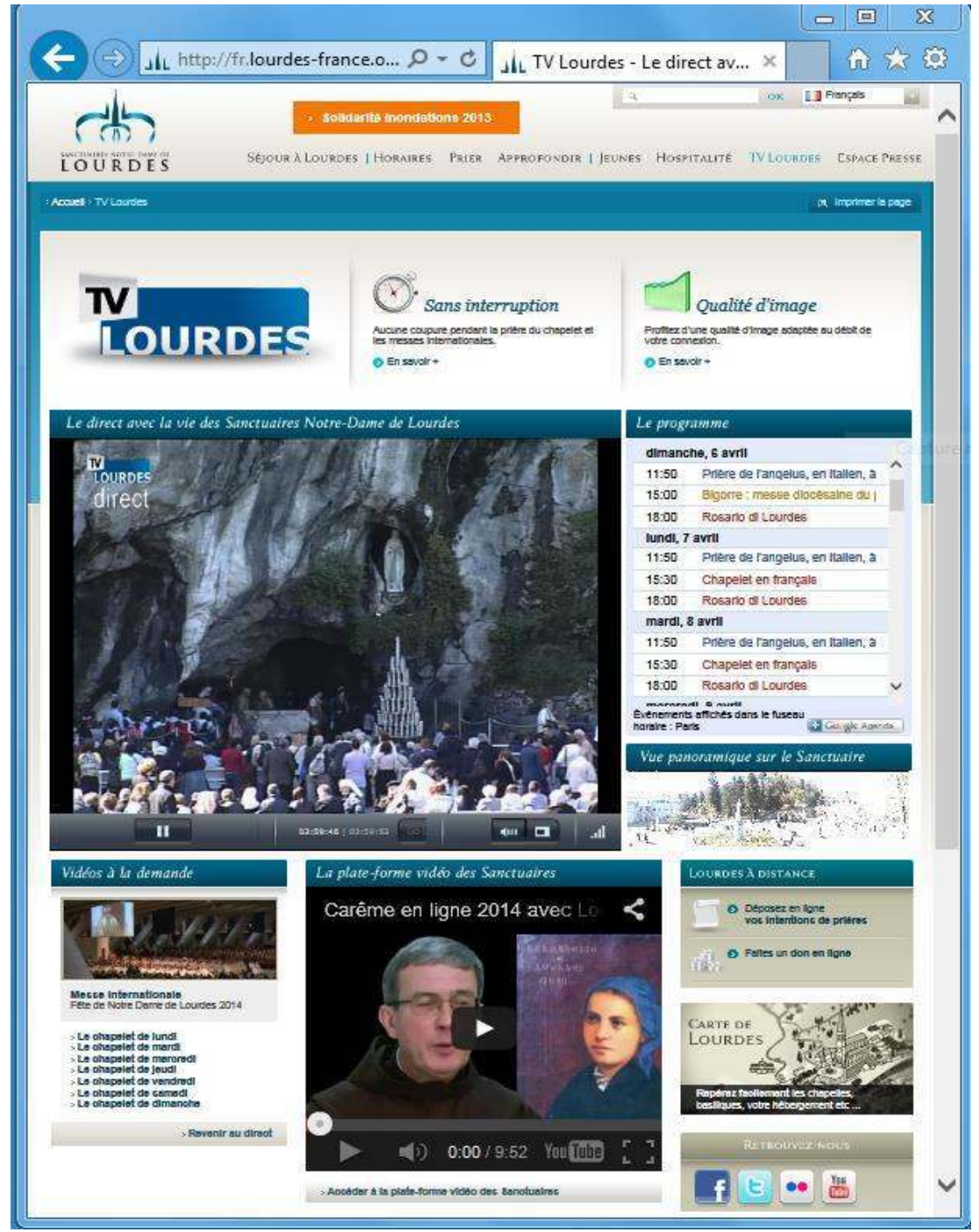

Figure 1 : Écran d'accueil de TV Lourdes, avec l'image retransmise de la grotte, principale source d'attraction des internautes. 


\section{Références bibliographiques}

BABIN P., 1999, Église et communications sociales en l'an 2000, <http://www.cccb.ca/site/frc/membres/assembleesplenieres-annuelles/72-assemblee-pleniere-1999/1921-thechurch-and-social-communications-by-rev-pierre-babin-omi>, dernière consultation le 5 septembre 2015.

BATESON G., 1977, Vers une écologie de l'esprit, tome 1, trad. par F. Drosso, L. Lot, E. Simion, Paris, Le Seuil.

BERNADOU P., 2008, Lourdes 1858-2008. Anthropologie de la communication d'un fait religieux et de ses médiatisations, thèse de doctorat en sciences de l'information et de la communication, sous la direction de Claude Nosal, Université Paul-Verlaine, Metz, 4 vol.

BERNARD A., 2013, "Le pèlerinage aujourd'hui : entre socialité et hagiographie ", Questions de communication, $\mathrm{n}^{\circ} 23$, pp.57-78.

BERNARD-WEIL É., 2002, Stratégies paradoxales en biomédecine et sciences humaines, Paris, L'Harmattan.

BROUWET N., 2014, 5 catéchèses sur l'évangélisation pour le diocèse de Tarbes et Lourdes, Association diocésaine de Tarbes et Lourdes, p.75.

CAILLEAUX M., 2007, Les médias des sanctuaires NotreDame de Lourdes (1958-2008) : éléments pour une histoire de la communication à Lourdes, Université de Pau et des Pays de l'Adour, mémoire de master en histoire contemporaine, sous la direction de Sylvaine Guinle-Lorinet.

CATELLANI A., 2014, "Prier en ligne à partir d'images: observations sémiotiques sur le site Notre Dame du Web", Médiation et Information. Religion \& Communication, $\mathrm{n}^{\circ} 38$, pp.101-111.

DOUYĖRE D., 2013, « L'Incarnation comme communication, ou l'auto-communication de Dieu en régime chrétien ", Questions de communication, $\mathrm{n}^{\circ} 23$, pp.31-55.

DUPRONT A., 1983, "Pèlerinages et lieux sacrés", Encyclopædia Universalis, $2^{\mathrm{e}}$ éd., 1989, Paris, vol.17, pp.790804

tic\&société - 9 (1-2), 2015 
GADRAS S. et I. PAILLART, 2013, " Les territoires et les médias dans la construction de l'espace public », dans J. NOYER, B. RAOUL et I. PAILLART (dir), Médias et territoires, l'espace public entre communication et imaginaire territorial, Villeneuve d'Ascq, Presses universitaires du Septentrion, pp.23-38.

GUINLE-LORINET S., 1993, Pierre-Marie Théas, un évêque à la rencontre $d u X X^{e}$ siècle, Tarbes-Toulouse, AGM-GRHI.

GUINLE-LORINET S. et P. BERNADOU, 2013, « La photographie au service de l'Eglise. Images de la grotte de Lourdes (1858-2010)", Communication, vol.31, $\mathrm{n}^{\circ} 1$, $<$ http://communication.revues.org/3856>, dernière consultation le 5 septembre 2015.

HENNIN B. de, 1993, "Schismogenèse », dans L. SFEZ (dir), Dictionnaire critique de la communication, Paris, PuF.

KAUFMAN S K., 2005, Consuming Visions. Mass Culture and the Lourdes Shrine, Ithaca, Londres, Cornell University Press.

LATOUR B., 1997, Nous n'avons jamais été modernes, essai d'anthropologie symétrique, Paris, La Découverte \& Syros.

LAURENTIN R., 1957-1966, Lourdes. Dossier des documents authentiques, 7 volumes, Paris, Lethielleux.

LAURENTIN R., 1978, Visage de Bernadette. I. Présentation, II. Album, Paris-Lourdes, Lethielleux / OEuvre de la Grotte.

MELOT A.-M., 1998, « Métacognition », dans O. HOUDE et al., Vocabulaire des sciences cognitives, Paris, PUF, pp.261263.

TERRENOIRE J.-P., 2006, «Sociologie visuelle. Études expérimentales de la réception. Les prolongements théoriques ou méthodologiques. ", Communications, vol.80, $\mathrm{n}^{\circ} 1$, pp.121143.

TURNER V., 1990, Le phénomène rituel. Structure et contrestructure [1969], trad. G. Guillet, Paris, PUF.

TURNER V. et E. TURNER, 1978, Image and Pilgrimage in Christian Culture, New York, Columbia University Press. 